\title{
Analyzing Neural Activity and Connectivity Using Intracranial EEG Data with SPM Software
}

\section{AUTHOR(S):}

Sato, Wataru; Kochiyama, Takanori; Uono, Shota; Usui, Naotaka; Kondo, Akihiko; Matsuda, Kazumi; Usui, Keiko; Toichi, Motomi; Inoue, Yushi

\section{CITATION:}

Sato, Wataru ... [et al]. Analyzing Neural Activity and Connectivity Using Intracranial EEG Data with SPM Software. Journal of Visualized Experiments 2018, 140: e58187.

\section{ISSUE DATE:}

2018-10-30

URL:

http://hdl.handle.net/2433/236372

RIGHT:

発行元の許可を得て掲載しています。 


\section{TITLE:}

Analyzing Neural Activity and Connectivity Using Intracranial EEG Data with SPM Software

\section{AUTHORS \& AFFILIATIONS:}

Wataru Sato ${ }^{1+}$, Takanori Kochiyama ${ }^{2+}$, Shota Uono ${ }^{3}$, Naotaka Usui ${ }^{4}$, Akihiko Kondo ${ }^{5}$, Kazumi Matsuda $^{5}$, Keiko Usui ${ }^{6}$, Motomi Toichi ${ }^{7}$, and Yushi Inoue ${ }^{5}$

+ equal contributors

${ }^{1}$ Kokoro Research Center, Kyoto University, Sakyo, Kyoto, Japan. ${ }^{2}$ Brain Activity Imaging Center, Advanced Telecommunications Research Institute International, Seika, Soraku, Kyoto, Japan. ${ }^{3}$ Department of Neurodevelopmental Psychiatry, Habilitation and Rehabilitation, Graduate School of Medicine, Kyoto University, Sakyo, Kyoto, Japan. ${ }^{4,5}$ National Epilepsy Center, Shizuoka Institute of Epilepsy and Neurological Disorders, Shizuoka, Japan. ${ }^{6}$

Department of System Neuroscience, Sapporo Medical University, Chuo, Sapporo, Japan. ${ }^{7}$ Faculty of Human Health Science, Graduate School of Medicine, Kyoto University, Sakyo, Kyoto, Japan.

Corresponding author:

Wataru Sato: sato.wataru.4v@kyoto-u.ac.jp

Naotaka Usui: n-usui@shizuokamind.org

\section{KEYWORDS:}

cross-frequency coupling; dynamic causal modeling (DCM); face; gamma oscillation; inferior occipital gyrus; intracranial electroencephalography (EEG); time-frequency analysis.

\section{SUMMARY:}

We present two analytical protocols that can be used to analyze intracranial electroencephalography data using the Statistical Parametric Mapping (SPM) software: timefrequency statistical parametric mapping analysis for neural activity, and dynamic causal modeling of induced responses for intra- and inter-regional connectivity.

\footnotetext{
ABSTRACT

Measuring neural activity and connectivity associated with cognitive functions at high spatial and temporal resolutions is an important goal in cognitive neuroscience. Intracranial electroencephalography (EEG) can directly record electrical neural activity and has the unique potential to accomplish this goal. Traditionally, averaging analysis has been applied to analyze intracranial EEG data; however, several new techniques are available for depicting neural activity and intra- and inter-regional connectivity. Here, we introduce two analytical protocols we recently applied to analyze intracranial EEG data using the Statistical Parametric Mapping (SPM) software: time-frequency SPM analysis for neural activity and dynamic causal modeling of induced responses for intra- and inter-regional connectivity. We report our analysis of intracranial EEG data during the observation of faces as representative results. The results revealed that the inferior occipital gyrus (IOG) showed gamma-band activity at very early stages (110 ms) in response to faces, and both the IOG and amygdala showed rapid intra- and interregional connectivity using various types of oscillations. These analytical protocols have the
} 
potential to identify the neural mechanisms underlying cognitive functions with high spatial and temporal profiles.

\section{INTRODUCTION}

Measuring neural activity and connectivity associated with cognitive functions at high spatial and temporal resolutions is one of the primary goals of cognitive neuroscience. However, accomplishing this goal is not easy. One popular method used to record neural activity is functional magnetic resonance imaging (MRI). Although functional MRI offers several advantages, such as a high spatial resolution at the millimeter level and non-invasive recording, a clear disadvantage of functional MRI is its low temporal resolution. In addition, functional MRI measures blood-oxygen-level-dependent signals, which only indirectly reflect electric neural activity. Popular electrophysiological methods, including electroencephalography (EEG) and magnetoencephalography (MEG), have high temporal resolutions at the millisecond level. However, they have relatively low spatial resolutions, because they record electric/magnetic signals at the scalp and must solve difficult inverse problems to depict brain activity.

Intracranial EEG can directly record electrical neural activity at high temporal (millisecond) and spatial (centimeter) resolutions ${ }^{1}$. This measure can provide valuable opportunities to understand neural activity and connectivity, although it has clear limitations (e.g., measurable regions are restricted to clinical criteria). Several intracranial EEG studies have applied traditional averaging analysis to depict neural activity. Although averaging analysis can sensitively detect time-locked and low-frequency band activation, it cannot detect non-phaselocked and/or high-frequency (e.g., gamma band) activation. In addition, functional neural coupling has not been analyzed in depth in the literature on intracranial EEG recordings. Several new techniques have been recently developed to depict neural activity and intra- and interregional connectivity in functional $\mathrm{MRI}$ and $\mathrm{EEG} / \mathrm{MEG}$ recordings, which can be applied to analyze intracranial EEG data.

Here, we introduce analytical protocols that we have recently applied to analyze intracranial EEG data using the Statistical Parametric Mapping (SPM) software. First, to reveal when, and at which frequency, the brain regions could be activated, we performed time-frequency SPM analysis ${ }^{2}$. This analysis decomposes the time and frequency domains simultaneously using a continuous wavelet transform and appropriately corrects the family-wise error (FWE) rate in the time-frequency maps using the random field theory. Second, to reveal how brain regions communicate, we applied dynamic causal modeling (DCM) of induced responses ${ }^{4}$. DCM enables the investigation of effective connectivity (i.e., the causal and directional influences among brain regions ${ }^{5}$ ). Although DCM was originally proposed as a tool for analyzing functional MRI data ${ }^{5}, \mathrm{DCM}$ of induced responses has been extended to analyze the time-varying power spectra of electrophysiological signals ${ }^{4}$. This analysis allows the depiction of both intra- and interregional neural connectivity. Several neurophysiological studies have suggested that local intraregional computations and long-range inter-regional communication mainly use gamma- and theta-band oscillations, respectively, and their interactions (e.g., entrainments) can be reflected by theta-gamma cross-frequency coupling ${ }^{3,6-8}$. This report focuses on the data analytical 
protocol; for an overview of background information $\mathrm{n}^{9,10}$ and recording protocols ${ }^{11}$ of intracranial EEG, please refer to the literature.

\section{PROTOCOL:}

Our study was approved by the local institutional ethics committee.

\section{Basic Information}

Note: The analytical protocols can be applied to various types of data without any restrictions as to specific participants, electrodes, reference methods, or electrode locations. In our example, we tested six patients suffering from pharmacologically intractable focal epilepsy. We tested patients who had no epileptic foci in the regions of interest.

1.1. Record intracranial EEG data during the cognitive experiment at the target electrodes. 1.1.1. Implant depth electrodes using the stereotactic method ${ }^{12}$.

1.1.2. Use subdural platinum electrodes (diameter: $2.3 \mathrm{~mm}$ ) and depth platinum electrodes (diameter: $0.8 \mathrm{~mm}$ ) to simultaneously measure cortical and subcortical activity, respectively. 1.1.3. Place reference electrodes on the surface of the skull of the midline dorsal frontal region, with the contacts of the electrodes facing away from the skull to avoid referential activation $^{12}$.

1.1.4. Amplify data, filter online (band pass: $0.5-300 \mathrm{~Hz}$ ), and sample at $1000 \mathrm{~Hz}$.

1.1.5. To record and statistically remove artifacts associated with eye movements, additionally record electrooculograms. Select the target electrodes based on theoretical interests. In addition, use individual MRI and computed tomography data to check the electrode locations.

\subsection{Sample and preprocess trial intracranial EEG data (Figure 1).}

Note: The analytical protocols can be applied to various types of data without any restriction to specific data-length or preprocessing methods.

1.2.1. Here, sample data during 3000 ms (pre-stimulus: 1000 ms; post-stimulus: 2000 ms) for each trial.

1.2.2. Because participants here showed abnormally high amplitude activity in some trials, possibly related to epilepsy, exclude these outlier trials using predefined thresholds ( $>800 \mu \mathrm{V}$ and $>5 S D$ ). Other preprocessing steps, including visual inspection and independent component analysis, may be required depending on the experimental objectives and conditions.

1.3. Convert the EEG system native format to a MATLAB-based SPM format (cf. SPM12 Manual 12.1 and 12.2).

Note: The most EEG data formats can be directly imported into SPM software by selecting Conversion in SPM Batch Editor interface and specifying all required input parameters. Another possible way is to use an example script "spm_eeg_convert_arbitrary_data.m" in the man/example_scripts subdirectory of the SPM program directory. This script provides a convenient way to convert an ASCII file or MAT file that can be exported by many EEG system with SPM format. 


\section{Time-Frequency SPM Analysis}

2.1. Set up SPM12 (http://www.fil.ion.ucl.ac.uk/spm/) and use the M/EEG analytical menu ${ }^{13}$ (Figure 2).

2.2. Perform the time-frequency SPM analysis ${ }^{2}$ by selecting Time-frequency analysis in the SPM menu for the preprocessed intracranial EEG data of each trial using continuous wavelet decomposition with Morlet wavelets based on predefined parameters (Figure 3).

Note: Wavelet transforms reveal the temporal evolution of spectral components by convolving intracranial EEG data with wavelets of multiple frequencies ${ }^{14}$.

2.2.1. Here, conduct wavelet decomposition using seven-cycle Morlet wavelets for the entire epoch (-1000-2000 ms) and frequency range of 4-300 Hz.

2.2.2. Determine the mother wavelet and number of cycles based on a previous study ${ }^{15}$. Note that the number of cycles in the wavelet controls the time-frequency resolutions and is recommended to be greater than 5 to ensure estimation stability ${ }^{13}$.

2.2.3. Determine time and frequency ranges based on the research interest.

2.3. Crop the resultant time-frequency maps automatically by selecting Crop in the SPM menu to remove edge effects. Here, crop the time-frequency maps into -200-500 ms.

2.4. Perform the data transformation (optional) and baseline correction by selecting Rescale TF in the SPM menu for the time-frequency maps to visualize the event-related power changes better and improve the normality of the data.

Note: Here, the time-frequency maps were log-transformed and baseline (-200-0 ms)corrected.

2.5. Convert the time-frequency maps into two-dimensional (2D) images by selecting Convert2Images in the SPM menu.

2.5.1. Smooth using a Gaussian kernel with a predefined full-width half-maximum (FWHM) value to compensate for inter-subject variability and conform to the assumptions of the random field theory used in the statistical inference ${ }^{2,13}$.

Note: Here, the time-frequency maps were smoothed with a Gaussian kernel of FWHM of 96 $\mathrm{ms}$ in the time domain and $12 \mathrm{~Hz}$ in the frequency domain based on a previous study ${ }^{2}$.

2.6. Enter the 2D images into the general linear model by selecting Specify 2 nd-level in the SPM menu.

2.7. Estimate the general linear model by selecting Model estimation in the SPM menu.

2.8. Perform statistical inferences on the time-frequency SPM $\{T\}$ data based on the random field theory ${ }^{2}$ by selecting Results in the SPM menu. Detect significantly activated timefrequency clusters with predefined thresholds (possibly corrected for multiple comparisons). Note: Here, the extent threshold of $p<0.05$, which was FWE-corrected for multiple comparisons, with a height threshold of $p<0.001$ (uncorrected) was used. 


\section{DCM of Induced Responses}

3.1. Set up SPM12 (http://www.fil.ion.ucl.ac.uk/spm/) and use the M/EEG analytical menu ${ }^{13}$ (Figure 4).

3.1.1. Start DCM analysis by clicking DCM button in the SPM menu. Activate DCM for induced responses by selecting IND in the list box. Import the preprocessed intracranial EEG data by clicking new data in the DCM for M/EEG menu.

3.2. Specify time window of interest, conditions of interest, contrasts for the selected conditions (this define the modulation inputs later used in network specification), frequency window of interest, and the number of the wavelet cycles in the DCM for M/EEG menu (Figure 5).

3.2.1. Use five-cycle Morlet wavelets (4-100 Hz in 1-Hz steps) and set the time window to 1$500 \mathrm{~ms}$.

3.2.2. Determine the wavelet cycle in accordance with the default setting. Note that the software recommendation is the value greater than 5 to ensure estimation stability ${ }^{13}$. The time-frequency ranges were determined based on our research interest. Note that a time window with an additional $\pm 512 \mathrm{~ms}$ was automatically used during computation to remove edge effects.

3.3. Based on the DCM framework ${ }^{4,5}$, define the (1) driving inputs, which represent the sensory inputs on neural states; (2) intrinsic connections, which embody the baseline connectivity among neural states and self-connections; and (3) modulatory effects on intrinsic connections via experimental manipulations for null and hypothesized models. Also define the type of modulation as linear (within-frequency) or nonlinear (between-frequency).

3.3.1. Specify intrinsic (linear and nonlinear) connections, driving inputs, and modulation inputs in the DCM for M/EEG menu.

3.3.2. Modify the default settings of some related parameters (e.g., prior stimulus onset time and duration) if necessary. Estimate the models by selecting invert DCM in the DCM for M/EEG menu. After that select Save results as img to save frequency-frequency modulatory coupling parameter images.

3.4. Conduct a random-effects Bayesian model selection (BMS) analysis ${ }^{17}$ by clicking BMS in the DCM for M/EEG menu to identify the optimal network model. Use the model expected probabilities and/or exceedance probabilities as evaluation measures.

3.5. Make inferences regarding the cross-frequency patterns of the modulatory connections using the winning model parameters by using the SPM menu (see Step 2).

3.5.1. Smooth the modulatory coupling parameter images by selecting Convert2Images in the SPM menu.

3.5.2. Perform general linear model analyses by selecting Specify 2 nd-level in the SPM menu.

3.5.3. Calculate the $2 \mathrm{D}$ SPM $\{T\}$ values by selecting Results in the SPM menu.

Note: Here, the FWHM was set at $8 \mathrm{~Hz}$ based on a previous study ${ }^{4}$. Significant values were exploratorily identified using a height threshold of $p<0.05$ (uncorrected). 


\section{REPRESENTATIVE RESULTS}

Using the protocol presented herein, we analyzed intracranial EEG data in response to faces ${ }^{18,19}$. We recorded data from six patients during the passive viewing of faces, mosaics, and houses in upright and inverted orientations. The contrasts of upright faces versus upright mosaics and upright faces versus upright houses revealed the face effect (i.e., face-specific brain activity relative to other objects). The contrast of upright faces versus inverted faces revealed the face-inversion effect (i.e., face-specific visual processing possibly related to configural/holistic processing $\left.{ }^{20}\right)$. As the target region for the time-frequency analysis and phase-amplitude cross-frequency coupling, we selected the right inferior occipital gyrus (IOG) based on previous neuropsychological ${ }^{21}$ and neuroimaging ${ }^{22}$ findings. For DCM, we tested the model in which the IOG and amygdala constitute a functional network during face processing based on previous anatomical evidence ${ }^{23}$.

\section{Time-frequency analysis}

Time-frequency analyses were conducted to investigate the temporal and frequency profiles of IOG activity during the processing of faces. Time-frequency maps were converted into 2D images and entered into the general linear model with the factors of stimulus type (face, house, and mosaic) and orientation (upright and inverted). Significant responses were identified using an FWE-corrected extent threshold of $p<0.05$ with a height threshold of $p<0.001$ (uncorrected). The contrasts testing the face effect (upright face vs. upright mosaic) consistently revealed significant rapid (110-500 ms) gamma-band activity (Figure 6a). The contrasts testing the face-inversion effect (upright face vs. inverted face) revealed significant gamma band activity at a later period (195-500 ms).

\section{DCM of induced responses}

DCM of induced responses was applied to test the functional network models of the IOG and amygdala. For both the face and face-inversion effects, the exceedance probabilities of the random-effects Bayesian model selection indicated that the model with intra-regional modulatory connectivity in both regions and bidirectional inter-regional modulatory connectivity was the most likely among all possible models (Figure 6b).

Next, we inspected the spectral profiles of the modulatory couplings in the best model. Significant effects were assessed for the entire spectral range with a height threshold of $p<$ 0.05 (uncorrected). Significant same- and cross-frequency modulatory couplings were observed for both intra- and inter-regional connectivity of both the face and face-inversion effects (Figure 6c). For example, as the intra-regional modulation of the face effect, the amygdala showed negative gamma-gamma same- and beta-gamma cross-frequency couplings. Meanwhile, the intra-IOG modulation showed a positive cross-frequency coupling in the theta/alpha/betagamma band. In addition, as the inter-regional coupling of the face effect, the IOG->amygdala modulation revealed a profile in which the theta/alpha band in the IOG facilitated the gamma band in the amygdala. For amygdala->IOG modulation, the gamma band in the amygdala inhibited the theta/alpha band and the same-frequency gamma band in the IOG. For the faceinversion effect, similar amygdala->IOG modulation, in which the gamma band in the amygdala 
inhibited the gamma band in the IOG, was observed. However, for IOG->amygdala modulation, the theta/alpha-gamma association observed in the face effect was not evident.

\section{DISCUSSION:}

The analytical protocols for intracranial EEG data using the SPM software introduced herein have several advantages compared with functional MRI. First, the protocols can depict neural activation at a high temporal resolution. Therefore, the results indicate whether the cognitive correlates of neural activation are implemented at early or late stages of processing. In our example, the face effect was identified during the very early stages (i.e., $110 \mathrm{~ms}$ ) of visual processing. In addition, the comparison of the temporal profiles of neural activity related to different psychological functions provide interesting implications. In particular, our example revealed different activation times for the face and face-inversion effects, beginning at $115 \mathrm{~ms}$ and $165 \mathrm{~ms}$, respectively, in the IOG. Such rich temporal information can deepen our understanding of neurocognitive mechanisms.

Furthermore, the protocols can depict intra- and inter-regional neural connectivity. Data from other neuroscientific measures, such as hemodynamic signals and scalp-recorded electromagnetic signals, contain a large amount of noise and require estimation based on several assumptions to extract the original electric signals, which can distort the resultant neural connectivity. Hence, the analysis of directly recorded electric signals is valuable. As an illustration, although our results revealed functional coupling between the IOG and amygdala during face processing, such coupling was not detected in a previous analysis of functional MRI data ${ }^{24}$. Understanding neural mechanisms requires the identification of causal relationships among neural circuits, which requires temporal information of neural activation.

However, it is important to note that the optimal protocol used to record and analyze intracranial EEG remains debated. For example, methodological studies have suggested that reference electrodes can pick up bodily physiological artifacts (e.g., eye movements and muscle activity) and environmental noise, and the suitable position of reference electrodes for intracranial EEG remains to be determined ${ }^{25,26}$. Several preprocessing methods (e.g., filtering and non-linear transformations) to remove artifacts (e.g., epileptic activity) have been proposed, although they are under debate ${ }^{27}$. A study also reported that time-frequency analyses using wavelet decomposition could blur the peaks in the original data ${ }^{28}$, and alternative analytical methods, such as the Hilbert-Huang transform, may offer better temporal resolutions ${ }^{29}$. The extraction of the high-frequency range may also be improved using such methods ${ }^{30}$. It has been noted that cross-frequency coupling could be biased by sharp non-linear transients and controlling for such confounding effects is needed ${ }^{31-33}$. Several different analytical methods have been proposed for the analysis of intra- and inter-regional coupling, such as the phase-locking value ${ }^{16}$, weighted phase lag index ${ }^{34}$, and Grander causality ${ }^{35}$, and it remains unclear which analyses and parameters (e.g., frequency) are the most relevant to cognitive processing ${ }^{3}$. In some cases, intracranial EEG data may not satisfy parametric assumptions and non-parametric analyses may be optimal ${ }^{36}$. Recently, other analytical protocols have been proposed ${ }^{37}$; compared with other protocols, those based on the SPM software may have the unique potential to provide a unified framework for the analysis of 
various types of neuroscientific data ${ }^{38}$. Researchers should pay close attention to advancements in analytical protocols for intracranial EEG data.

In summary, we introduced analytical protocols that we recently applied to analyze intracranial EEG data, which include time-frequency SPM analysis, cross-frequency coupling, and DCM of induced responses. We believe these analytical protocols can identify neural correlates of cognitive functions with high spatial and temporal profiles.

\section{ACKNOWLEDGMENTS:}

This study was supported by funds from the Benesse Corporation, Japan Society for the Promotion of Science (JSPS) Funding Program for Next Generation World-Leading Researchers (LZO08), the Organization for Promoting Research in Neurodevelopmental Disorders, and the JSPS KAKENHI (15K04185; 18K03174).

\section{DISCLOSURES:}

The authors have nothing to disclose.

\section{REFERENCES:}

1. Lachaux, J.P., Rudrauf, D., Kahane, P. Intracranial EEG and human brain mapping. Journal of Physiology - Paris. 97 (4-6), 613-628 (2003).

2. Kilner, J.M., Kiebel, S.J., Friston, K.J. Applications of random field theory to electrophysiology. Neuroscience Letters. 374 (3), 174-178 (2005).

3. Canolty, R.T., Knight, R.T. The functional role of cross-frequency coupling. Trends in Cognitive Sciences. 14 (11), 506-515 (2010).

4. Chen, C.C. et al. A dynamic causal model for evoked and induced responses. Neuroimage. 59 (1), 340-348 (2012).

5. Friston, K.J., Harrison, L., Penny, W. Dynamic causal modelling. Neuroimage. 19 (4), 1273-1302 (2003).

6. Canolty, R.T. et al. High gamma power is phase-locked to theta oscillations in human neocortex. Science. 313, 1626-1628 (2006).

7. Tort, A.B. et al. Dynamic cross-frequency couplings of local field potential oscillations in rat striatum and hippocampus during performance of a T-maze task. Proceedings of the National Academy of Sciences of the United States of America, 105 (51), 20517-20522 (2008). 8. Voytek, B. et al. Shifts in gamma phase-amplitude coupling frequency from theta to alpha over posterior cortex during visual tasks. Frontiers in Human Neuroscience. 4, 191 (2010). 9. Mukamel, R., Fried, I. Human intracranial recordings and cognitive neuroscience. Annual Review of Psychology, 63, 511-537 (2012).

10. Parvizi, J., Kastner, S. Promises and limitations of human intracranial electroencephalography. Nature Neuroscience, 21, 474-483 (2018).

11. Hill, N.J. et al. Recording human electrocorticographic (ECOG) signals for neuroscientific research and real-time functional cortical mapping. Journal of Visualized Experiments, 64, 3993 (2012).

12. Herrmann, C.S., Rach, S., Vosskuhl, J., Strüber, D. Time-frequency analysis of eventrelated potentials: A brief tutorial. Brain Topography, 27 (4), 438-450 (2014). 
13. Litvak, V. et al. EEG and MEG data analysis in SPM8. Computational Intelligence and Neuroscience. 2011, 852961 (2011).

14. Mihara, T., Baba, K. Combined use of subdural and depth electrodes. Epilepsy Surgery (second ed.). (pp. 613-621) (2001).

15. Kilner, J., Bott, L., Posada, A. Modulations in the degree of synchronization during ongoing oscillatory activity in the human brain. European Journal of Neuroscience. 21, 25472554 (2005).

16. Lachaux, J.P., Rodriguez, E., Martinerie, J., Varela, F.J. Measuring phase synchrony in brain signals. Human Brain Mapping. 8 (4), 194-208 (1999).

17. Stephan, K.E., Penny, W.D., Daunizeau, J., Moran, R.J., Friston, K.J. Bayesian model selection for group studies. Neuroimage. 46 (4), 1004-1017 (2009).

18. Sato, W. et al. Rapid, high-frequency, and theta-coupled gamma oscillations in the inferior occipital gyrus during face processing. Cortex. 60, 52-68 (2014).

19. Sato, W. et al. Bidirectional electric communication between the inferior occipital gyrus and the amygdala during face processing. Human Brain Mapping. 38 (2), 4511-4524 (2017).

20. Bartlett, J.C, Searcy, J., Abdi, H. What are the routes to face recognition? Perception of faces, objects, and scenes: Analytic and holistic processing (pp. 21-52) (2003).

21. Bouvier, S.E, Engel, S.A. Behavioral deficits and cortical damage loci in cerebral achromatopsia. Cerebral Cortex 16 (2), 183-191 (2006).

22. Pitcher, D., Walsh, V., Duchaine, B. The role of the occipital face area in the cortical face perception network. Experimental Brain Research. 209 (4), 481-493 (2011).

23. Latini, F. New insights in the limbic modulation of visual inputs: The role of the inferior longitudinal fasciculus and the Li-Am bundle. Neurosurgical Review. 38 (1), 179-189 (2015).

24. Davies-Thompson, J., Andrews, T.J. Intra- and interhemispheric connectivity between face-selective regions in the human brain. Journal of Neurophysiology. 108 (11), 3087-3095 10.1152/jn.01171.2011 (2012).

25. Jerbi, K. et al. Saccade related gamma-band activity in intracerebral EEG: dissociating neural from ocular muscle activity. Brain Topography. 22, 18-23 (2009).

26. Buzsáki, G., Silva, F.L. High frequency oscillations in the intact brain. Progress in Neurobiology, 98, 241-249 (2012).

27. Benayoun, M., Kohrman, M., Cowan, J., van Drongelen, W. EEG, temporal correlations, and avalanches. Journal of Clinical Neurophysiology, 27 (6), 458-464 10.1097/WNP.0b013e3181fdf8e5 (2010).

28. Herrmann, C.S., Grigutsch, M., Busch, N.A. EEG oscillations and wavelet analysis. Eventrelated potentials: A methods handbook. (pp. 229-259) (2005).

39. Pigorini, A. et al. Time-frequency spectral analysis of TMS-evoked EEG oscillations by means of Hilbert-Huang transform. Journal of Neuroscience Methods. 198 (2), 236-245 10.1016/j.jneumeth.2011.04.013 (2011).

30. Holdgraf, C.R. et al. Rapid tuning shifts in human auditory cortex enhance speech intelligibility. Nature communications, 7, 13654 10.1038/ncomms13654 (2016).

31. Aru, J. et al. Untangling cross-frequency coupling in neuroscience. Current Opinion in Neurobiology, 31, 51-61 10.1016/j.conb.2014.08.002 (2015). 
32. Gerber, E.M., Sadeh, B., Ward, A., Knight, R.T., Deouell, L.Y. Non-sinusoidal activity can produce cross-frequency coupling in cortical signals in the absence of functional interaction between neural sources. PLoS One, 11 (12), e0167351 10.1371/journal.pone.0167351 (2016). 33. Cole, S.R., Voytek, B. Brain oscillations and the importance of waveform shape. Trends in Cognitive Sciences, 21 (2), 137-149 10.1016/j.tics.2016.12.008 (2017).

34. Mikulan, E. et al. Intracranial high- $\gamma$ connectivity distinguishes wakefulness from sleep. Neuroimage, 169, 265-277 10.1016/j.neuroimage.2017.12.015 (2018).

35. Zheng, J. et al. Amygdala-hippocampal dynamics during salient information processing. Nature communications, 8, 14413 10.1038/ncomms14413 (2017).

36. Maris, E., Oostenveld, R. Nonparametric statistical testing of EEG- and MEG-data. Journal of Neuroscience Methods, 164 (1), 177-190 (2007).

37. Stolk, A. et al., Integrated analysis of anatomical and electrophysiological human intracranial data. Nature Protocols, 13, 1699-1723 10.1038/s41596-018-0009-6 (2018). 38. Friston, K.J. et al. Dynamic causal modelling revisited. Neurolmage (in press). 
Raw EEG signals

$1000 \mathrm{~Hz}$ sampling rate

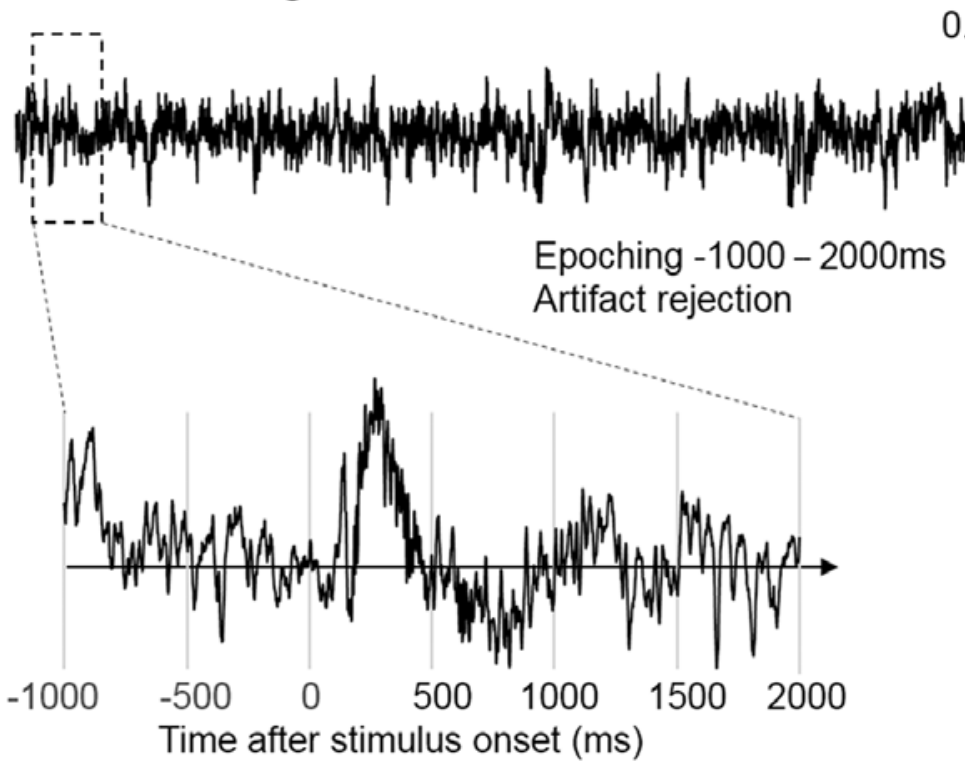

$0.5-300 \mathrm{~Hz}$ online filter

Figure 1. Illustration of the generation of the trial intracranial electroencephalography data. 


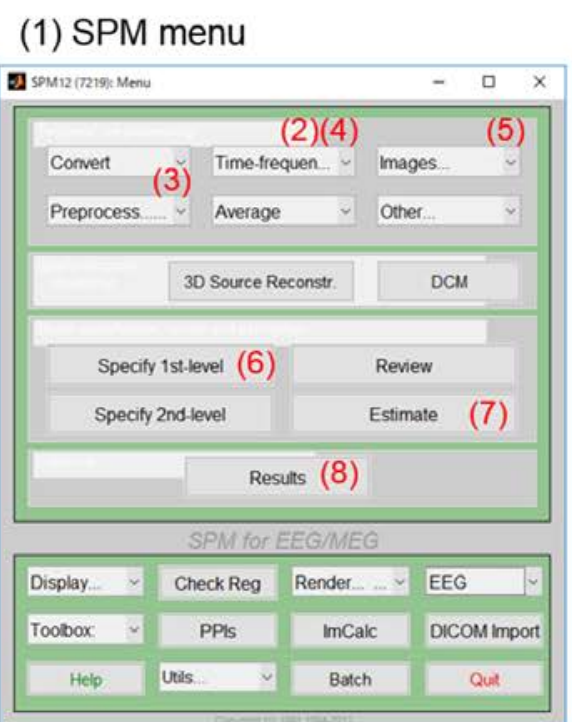

(4) Baseline correction
(2) Time-frequency analysis

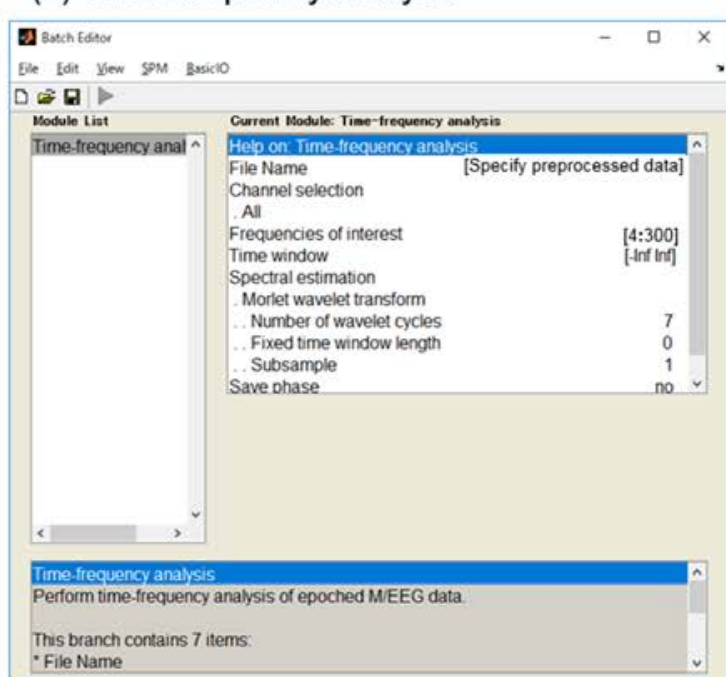

(5) Conversion

(3) Cropping

2 soth Esanor
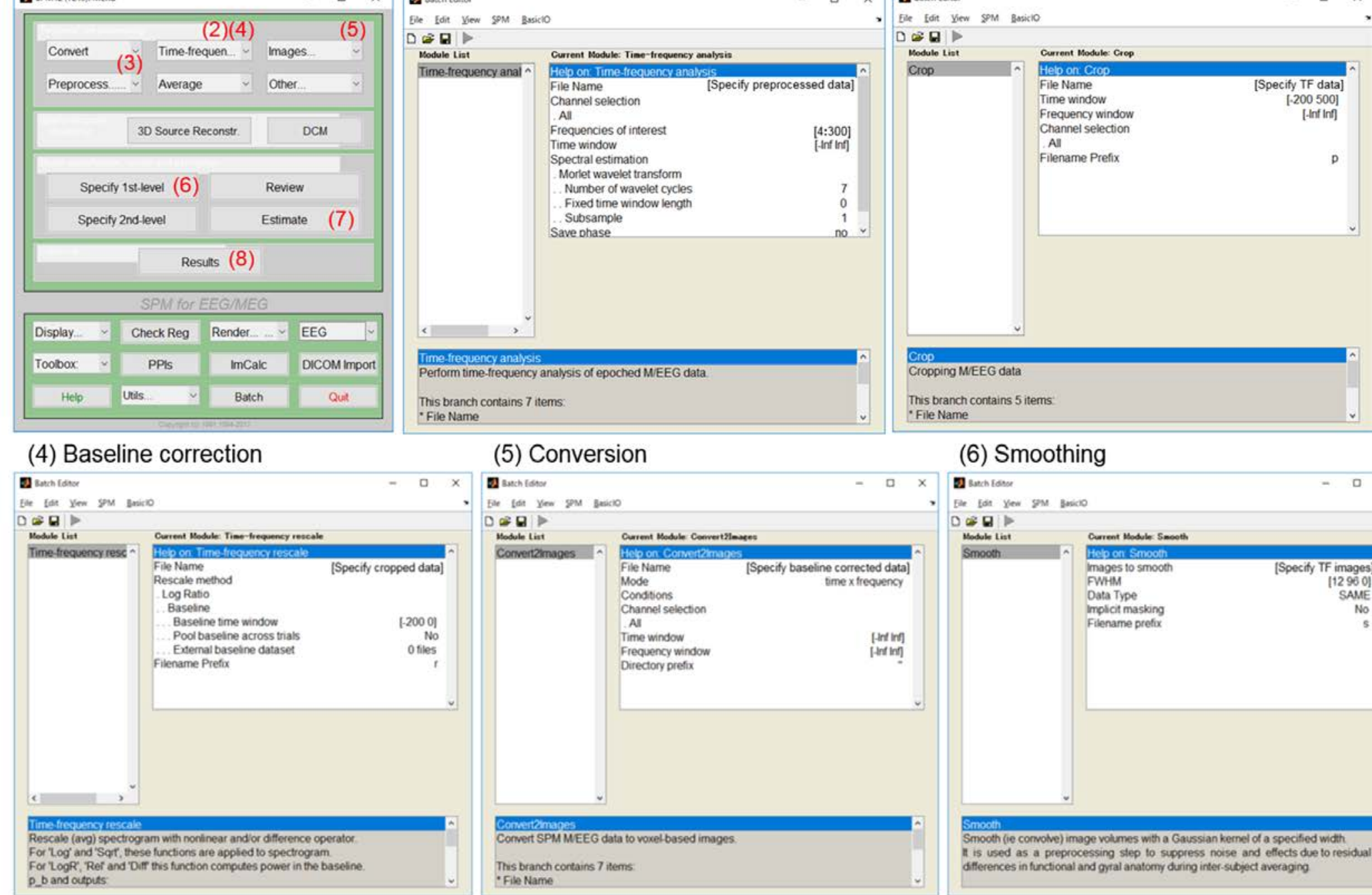

alisentand

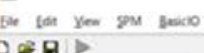

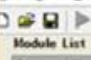

Comentarmages -

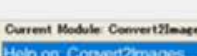
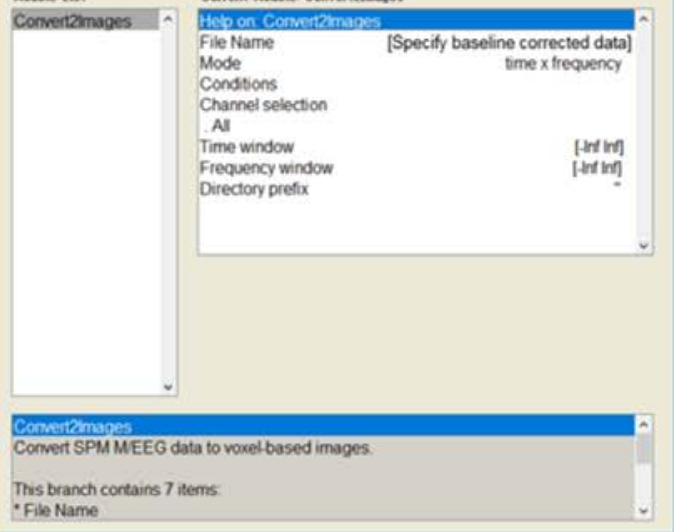

(6) Smoothing

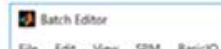

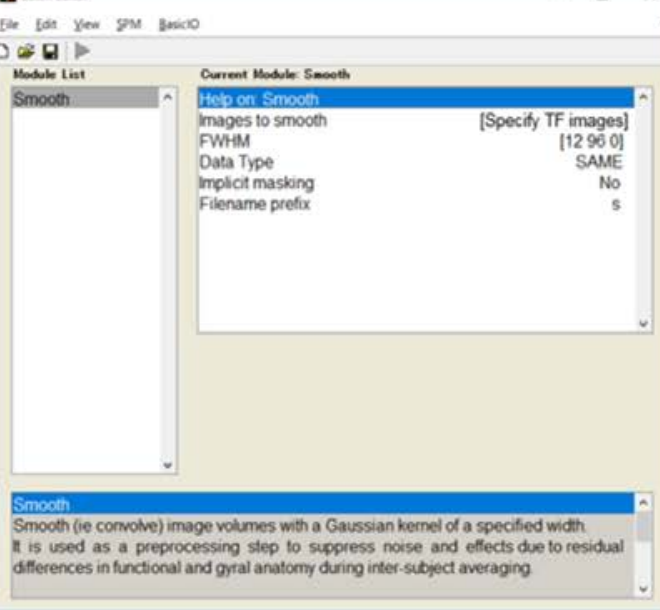

Figure 2. Graphic user interfaces of the Statistical Parametric Mapping (SPM) software for time-frequency analyses. (1) SPM menu. (2) Time-frequency analysis. (3) Cropping. (4) Baseline correction. (5) Conversion. (6) Smoothing. (7) Statistical model. (8) Model estimation. (9) Contrast. (10) Statistical inference. 
(7) Statistical model

\section{Q2 ostch tolitor}

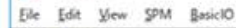

Nobute List

Factorial design spe -

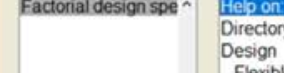

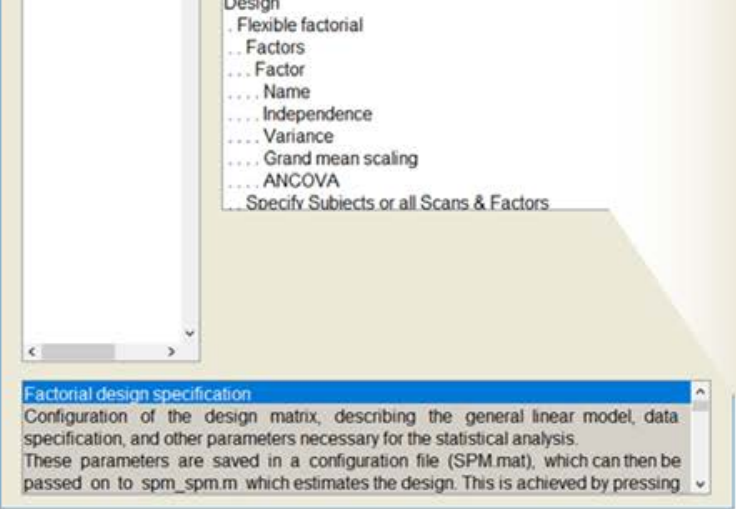

(8) Model estimation

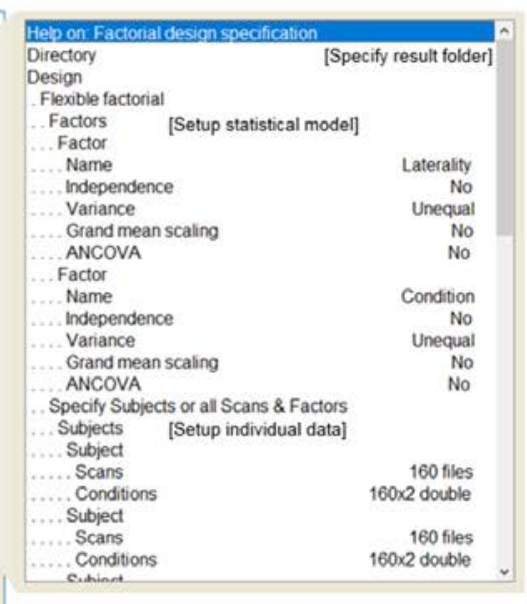

\section{(8) Model estimation}

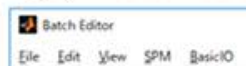

口乞口

Module List
Model estimation -

Current Hobule Model estieation

Heb on Model estimation
[Specify SPM.mat]

Write residuals

. Classical

, ,

Eodel estimation Likethood) or Bayesian algonithms After parameter estimation, the Resutts' button can be used to specify contrasts that
will produce Statsitical Parametric Maps (SPMs) or Posterior Probability Maps

\section{(9) Contrast}

SPM contrast manager

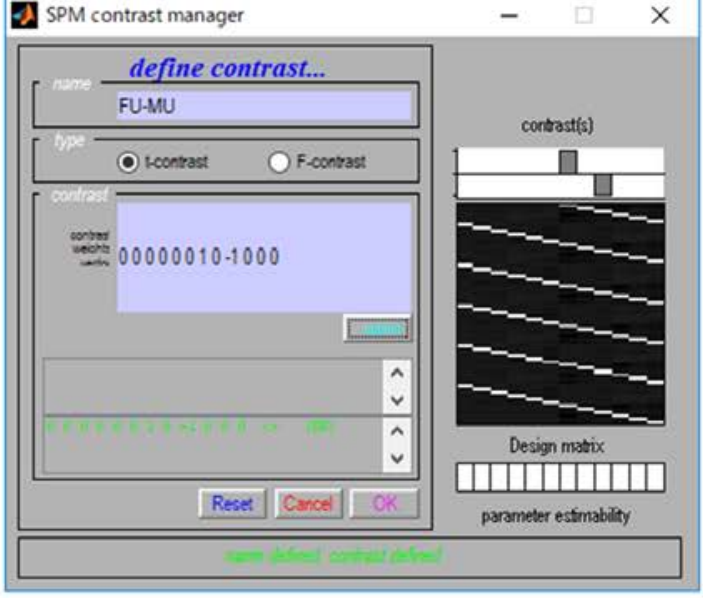

(10) Statistical inference

\begin{tabular}{|c|c|}
\hline SPM12 (7219): Stats: Results & $-\quad \square$ \\
\hline apply masking & \begin{tabular}{|c|c|} 
none) contras image atlas \\
\end{tabular} \\
\hline p value adjustment to control & none \\
\hline threshold $\{\pi$ or $p$ value\} & 0.001 \\
\hline \& extent threshold \{voxels\} & $\begin{array}{l}\text { [Input cluster size } \\
\text { (equivalent to } 0.05 \mathrm{FWE} \text {-corrected] }\end{array}$ \\
\hline Data Type: ... & v \\
\hline $\begin{array}{l}\text { Data Type: } \ldots \\
\text { Volumetric (2D/3D) } \\
\text { Scalp-Time } \\
\text { Scalp-Frequency }\end{array}$ & \\
\hline Time-Frequency & \\
\hline
\end{tabular}




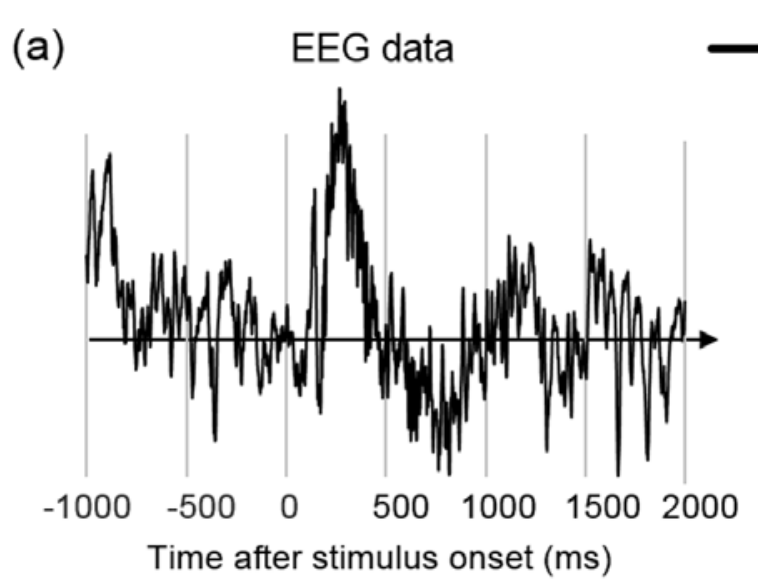

(b) Wavelet TF decomposition $\longrightarrow$ (c)

Cropped (-200 - 500ms), log-transformed

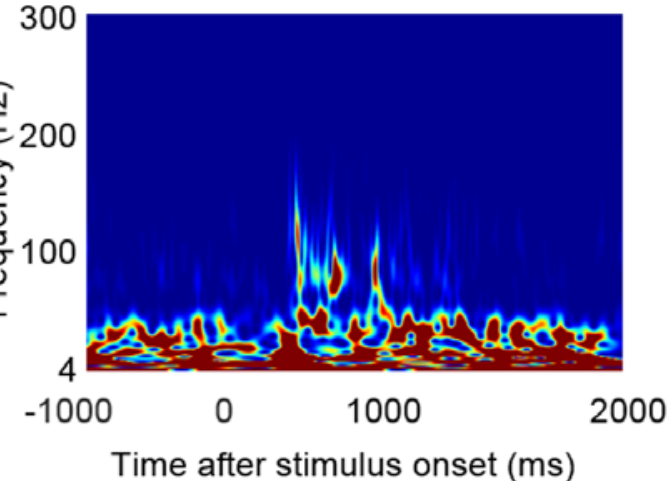
and baseline $(-200-0 \mathrm{~ms})$ corrected TF map

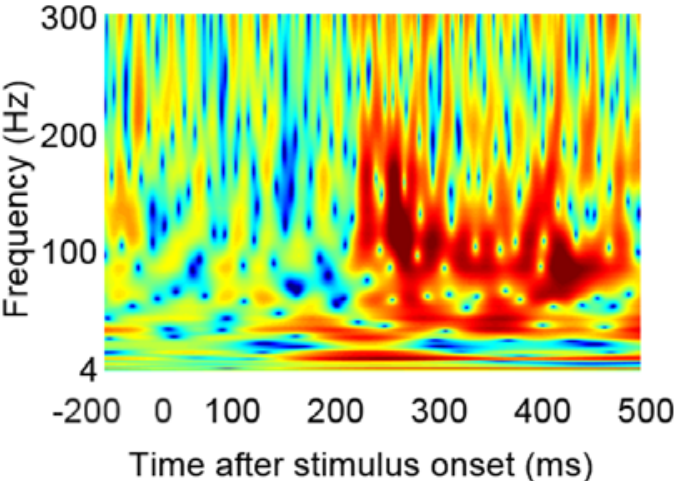

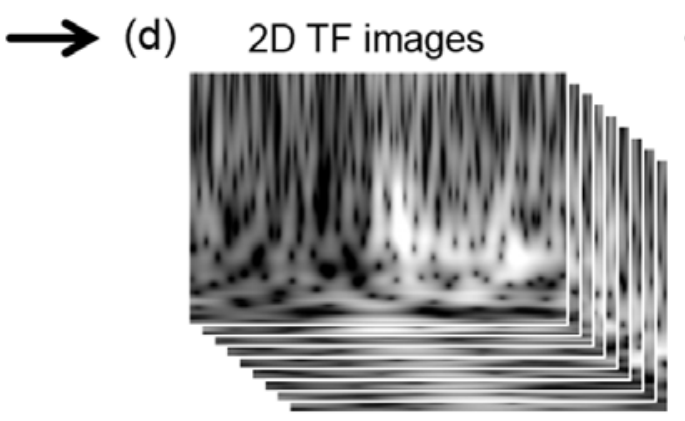

Data from all trials, conditions, and participants (e) Statistical model

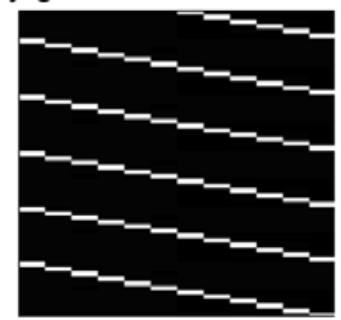

Design matrix

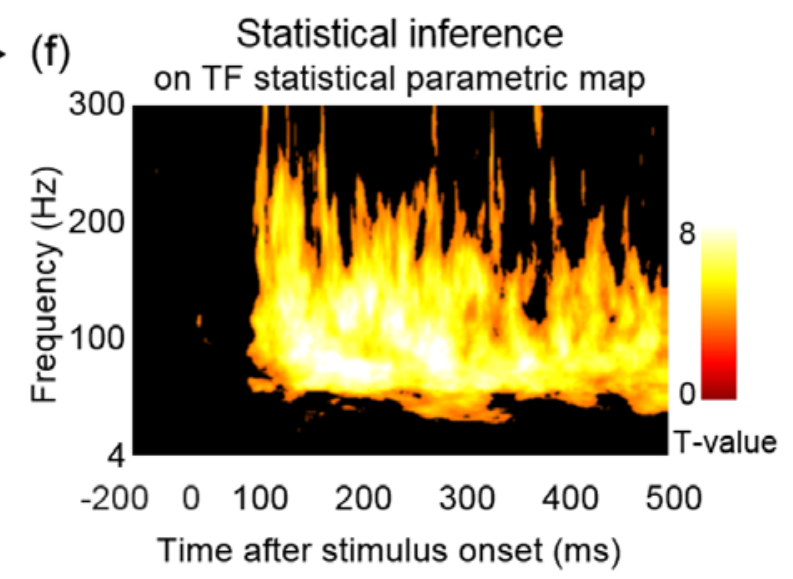

Figure 3. Flowchart of the time-frequency analysis using the Statistical Parametric Mapping (SPM) software. (a) Prepare the preprocessed intracranial electroencephalography (EEG) data of each trial. (b) Conduct time-frequency (TF) decomposition for the EEG data using continuous wavelet transform. (c) Crop, log-transform, and baseline correct for the TF maps. (d) Convert the TF maps into two-dimensional (2D) images. (e) Enter the 2D images into the general linear model. (f) Perform statistical inferences on the TF $\operatorname{SPM}\{T\}$ data. 
(1) SPM menu

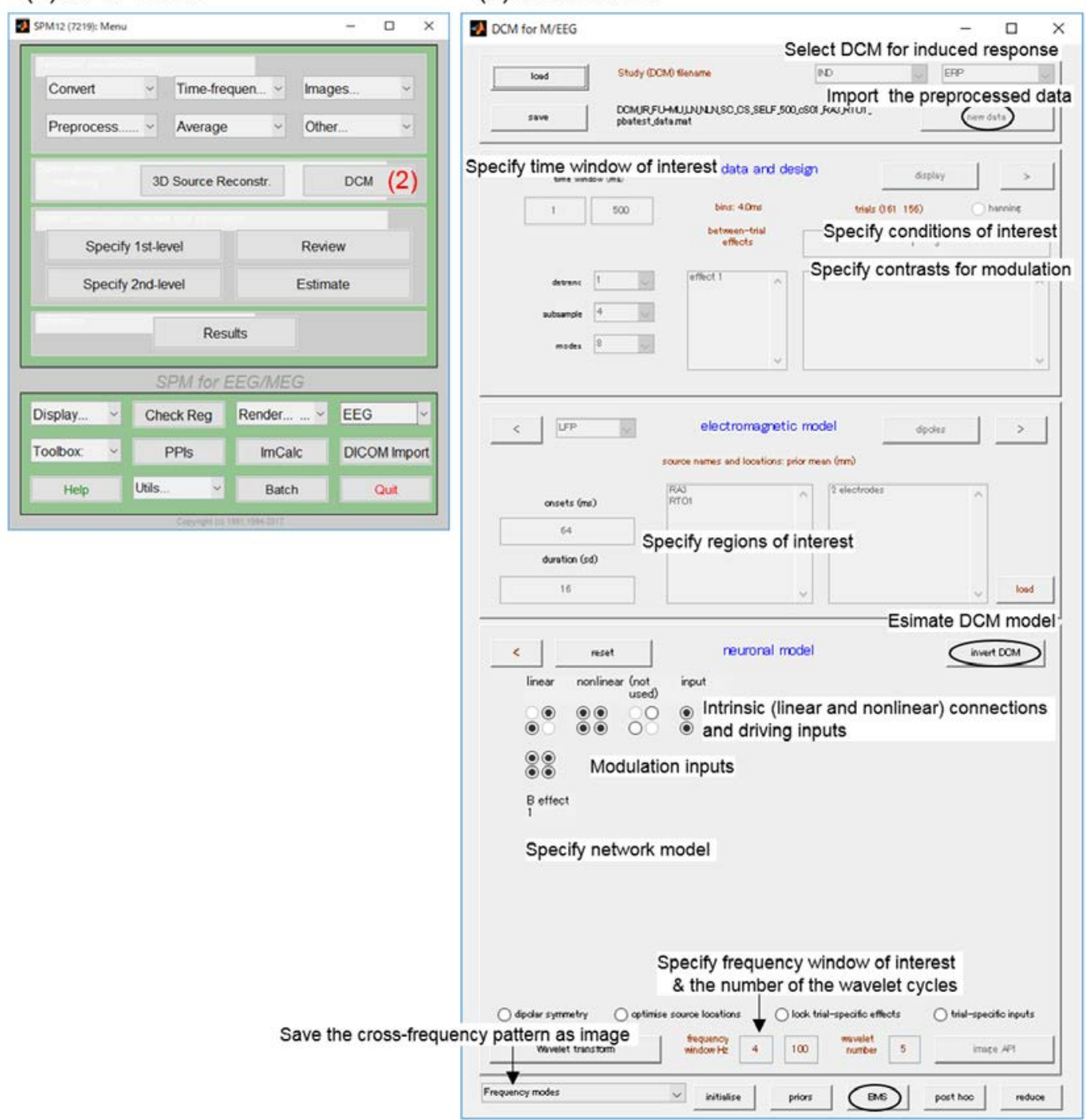

Figure 4. Graphic user interfaces of the Statistical Parametric Mapping (SPM) software for dynamic causal modeling (DCM) analyses. (1) SPM menu. (2) DCM menu. (3) Bayesian model selection. (4) Smoothing. (5) Statistical model. (6) Model estimation. (7) Contrast. (8) Statistical inference. 


\section{(3) Bayesian model selection}

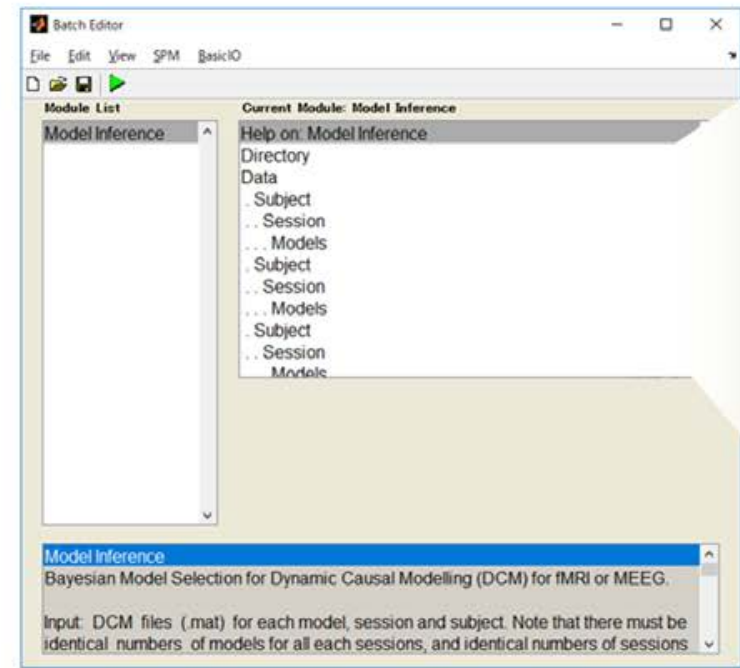

\section{(5) Statistical model}

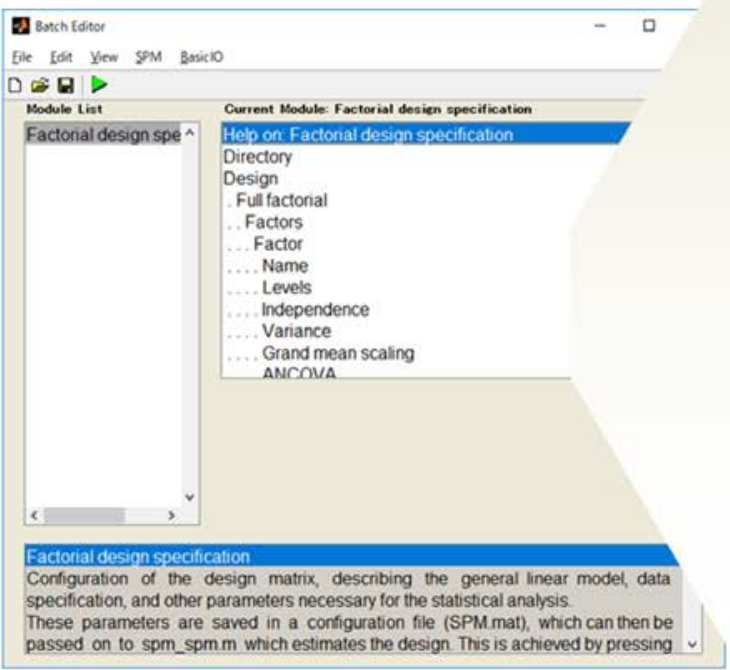

\section{(4) Smoothing}

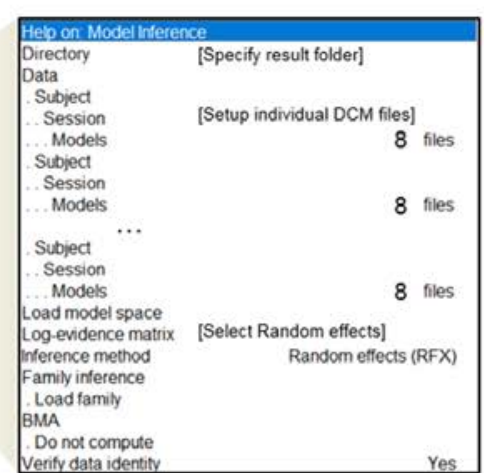

\begin{tabular}{|c|c|c|}
\hline \multicolumn{3}{|c|}{ Heh on Factorial dosing specafication } \\
\hline Directory & [Specity & sult folder] \\
\hline 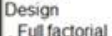 & & \\
\hline $\begin{array}{l}\text { Fulfaconal } \\
\text { Factors }\end{array}$ & [Setup statistical model] & \\
\hline Factor & & \\
\hline Name & & $\begin{array}{ll}B \mathrm{mbx} \\
4\end{array}$ \\
\hline $\begin{array}{l}\text { Levels } \\
\text { Independ }\end{array}$ & & $\begin{array}{l}4 \\
\text { No }\end{array}$ \\
\hline $\begin{array}{l}\text { Independ } \\
\text { Variance }\end{array}$ & & Unequal \\
\hline Grand $\mathrm{me}$ & scaling & $\begin{array}{ll}\text { Noteqal } \\
\text { No }\end{array}$ \\
\hline ANCOVA & & № \\
\hline Cells & [Setup individual data] & \\
\hline $\begin{array}{l}\text { Cell } \\
\text { Levels }\end{array}$ & & \\
\hline Scans & & 6 files \\
\hline & & \\
\hline $\begin{array}{l}\text { Levels } \\
\text { Scans }\end{array}$ & & $6{ }^{2}{ }^{2}$ \\
\hline Cell & & \\
\hline Levels & & $6{ }_{6 f l e s}^{3}$ \\
\hline Cetis & & \\
\hline Levels & & \\
\hline Scans & & 6 files \\
\hline Generate c & & Yes \\
\hline Mutiple covan & & \\
\hline Masking & & \\
\hline $\begin{array}{l}\text { Threshold m } \\
\text { None } \\
\end{array}$ & & \\
\hline - Impicict Mash & & Yes \\
\hline $\begin{array}{l}\text { Explict Mast } \\
\text { Globalcaliculat }\end{array}$ & & \\
\hline $\begin{array}{l}\text { Global calculat } \\
\text { Omit }\end{array}$ & & \\
\hline Global normal & & \\
\hline $\begin{array}{l}\text { Overall granci } \\
\text { Noo }\end{array}$ & an scaling & \\
\hline Normalisatio & & None \\
\hline
\end{tabular}

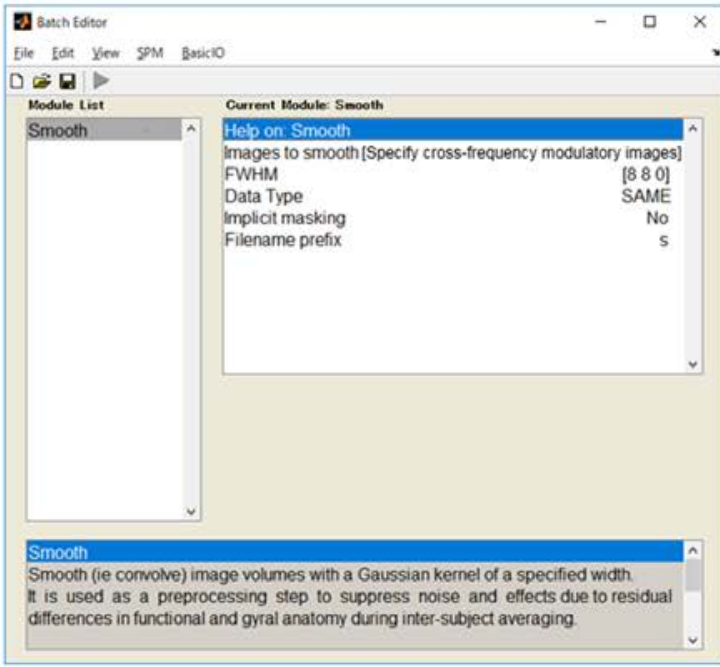

\section{(6) Model estimation}

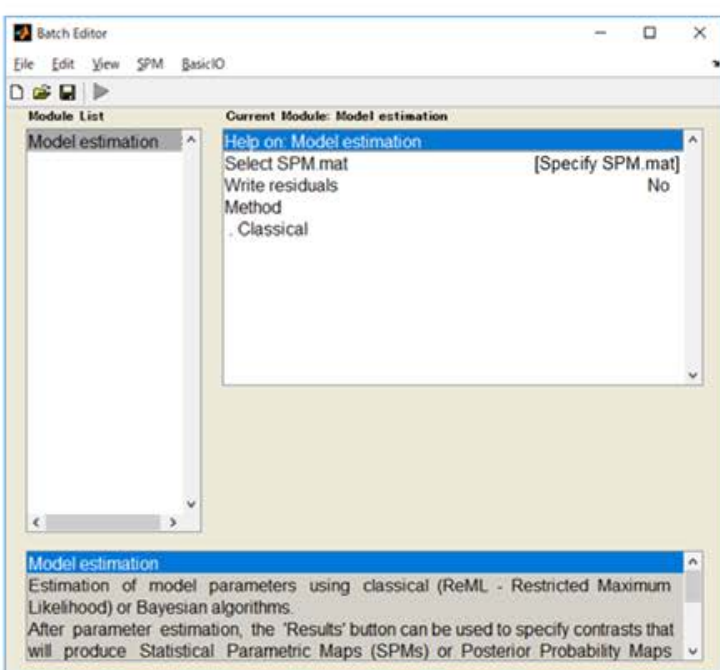




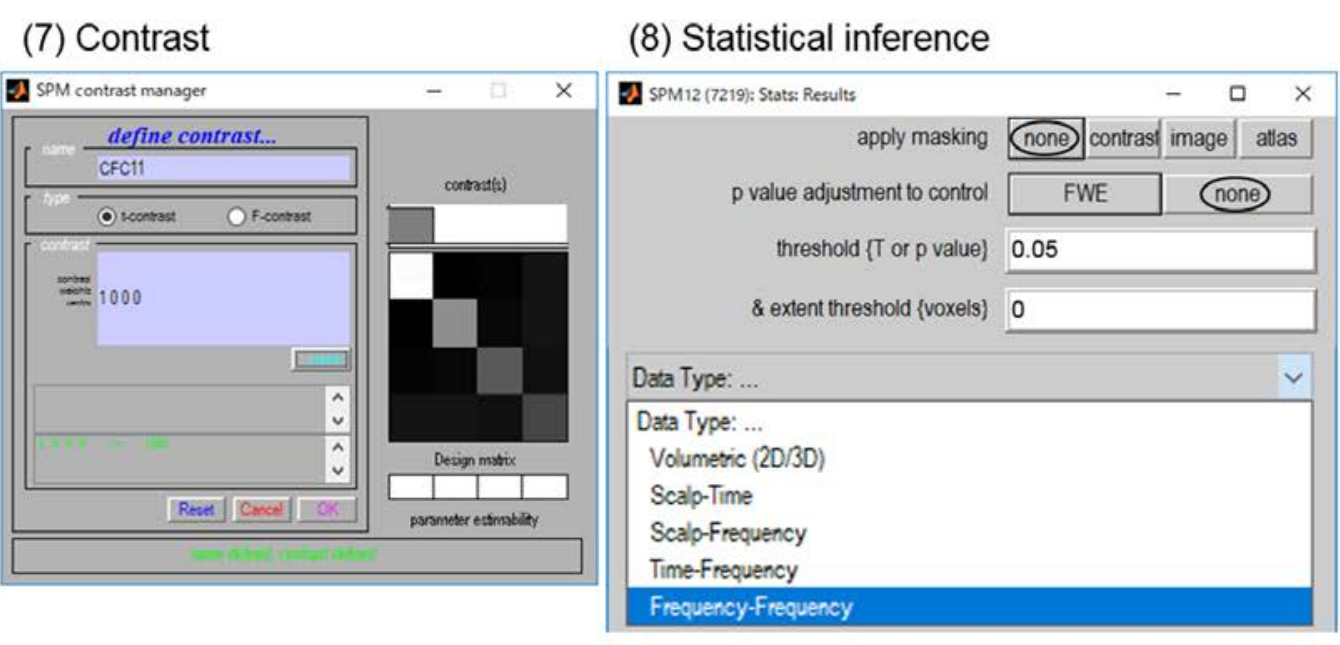



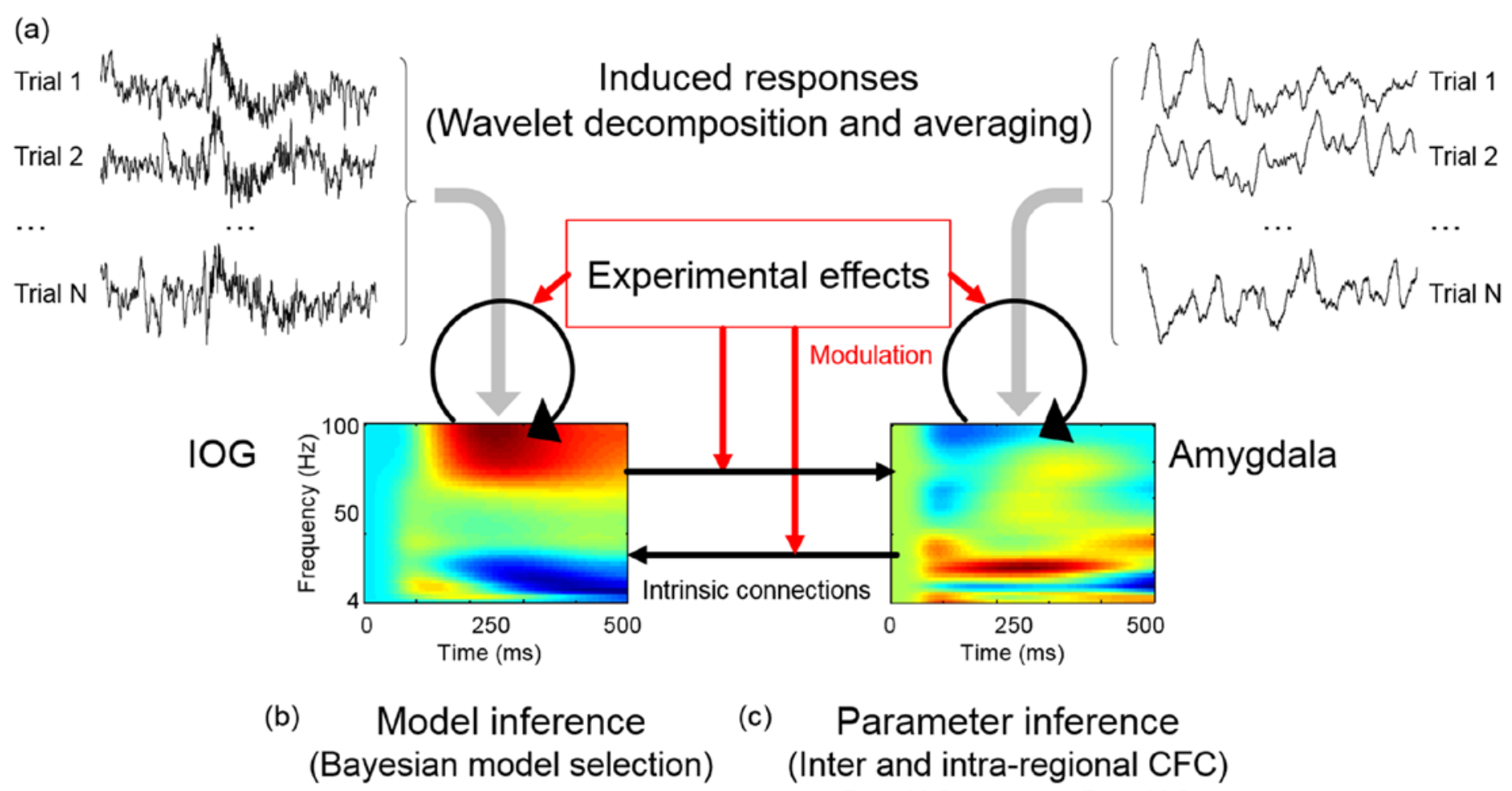

(c) Parameter inference

(Bayesian model selection)

\section{(Inter and intra-regional CFC)}
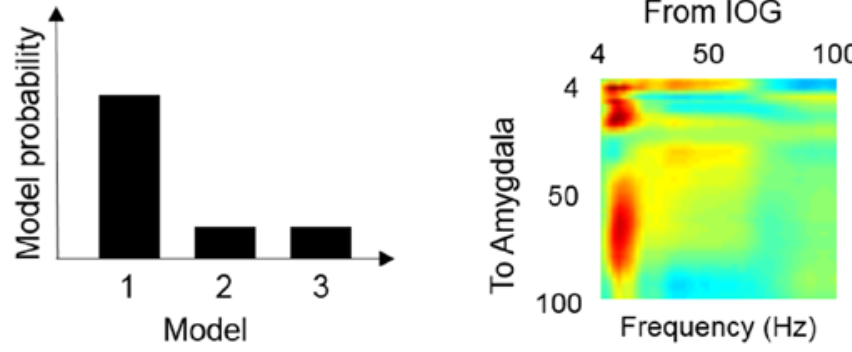

From IOG

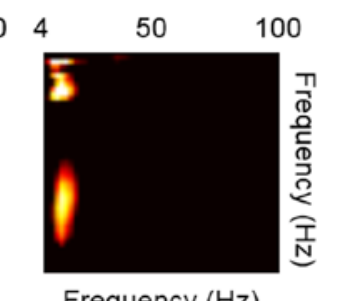

Frequency $(\mathrm{Hz})$

Figure 5. Flowchart of the dynamic causal modeling of induced responses. (a) Calculate the time-frequency spectra for each trial of the targeted multiple electrodes (the inferior occipital gyrus (IOG) and amygdala in our example) using continuous wavelet decomposition. Average the spectral magnitudes of time-frequency responses to yield the induced response. Then, define the driving input, intrinsic connections, and modulation of intrinsic connections by experimental manipulations. Construct models to test hypotheses and estimate the models. (b) Conduct a random-effects Bayesian model selection analysis to identify the optimal model. (c) Convert the frequency-frequency modulatory coupling parameters into two-dimensional (2D) images (with smoothing). Then, perform random-effects general linear model analyses and calculate the 2D SPM $\{T\}$ values. 
(a) Time-frequency analysis
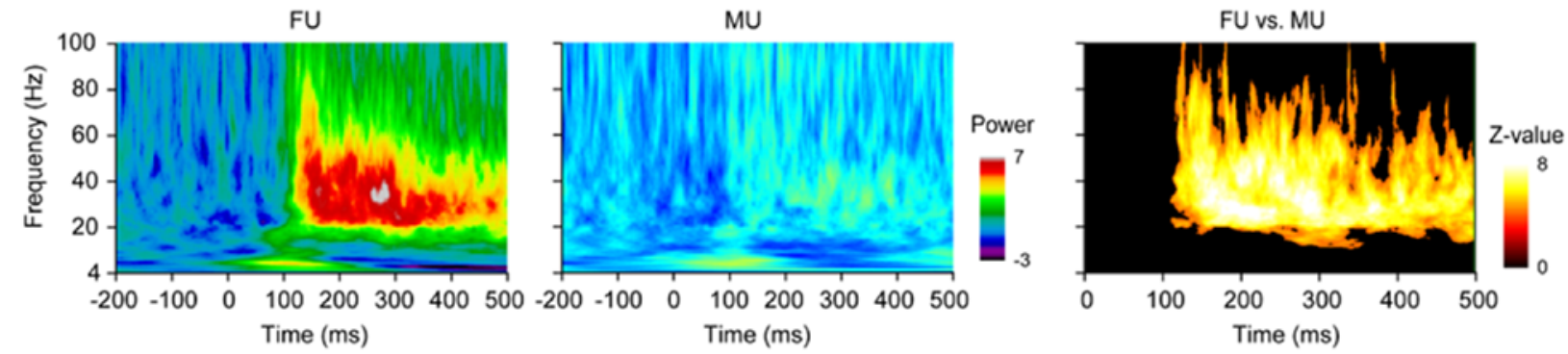

(b) DCM models
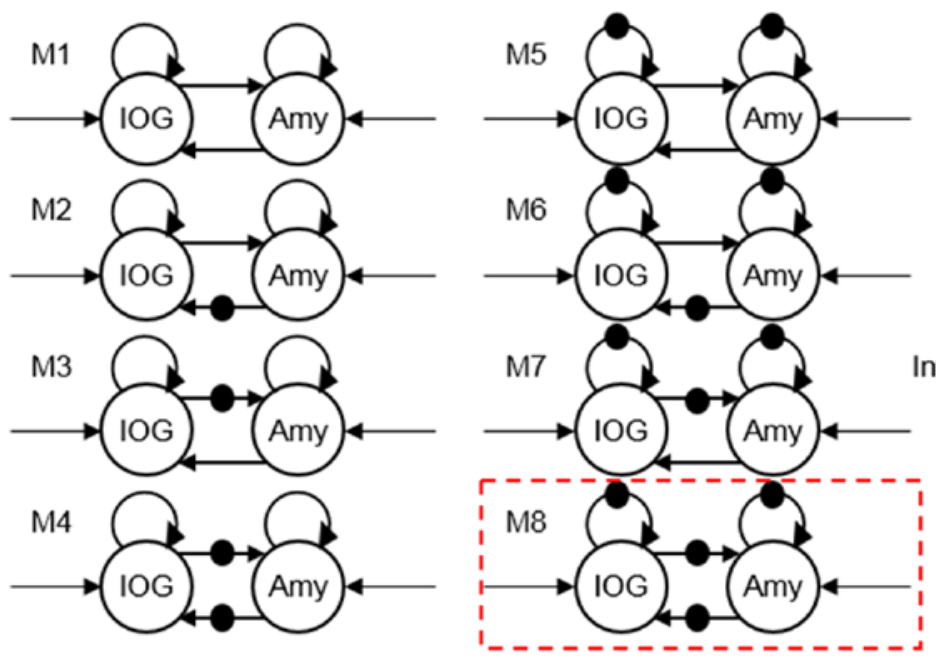

Intrinsic connection only

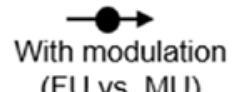

(FU vs. MU)

\section{Best model}

(d) Cross frequency coupling for modulatory connectivity (FU vs. MU)
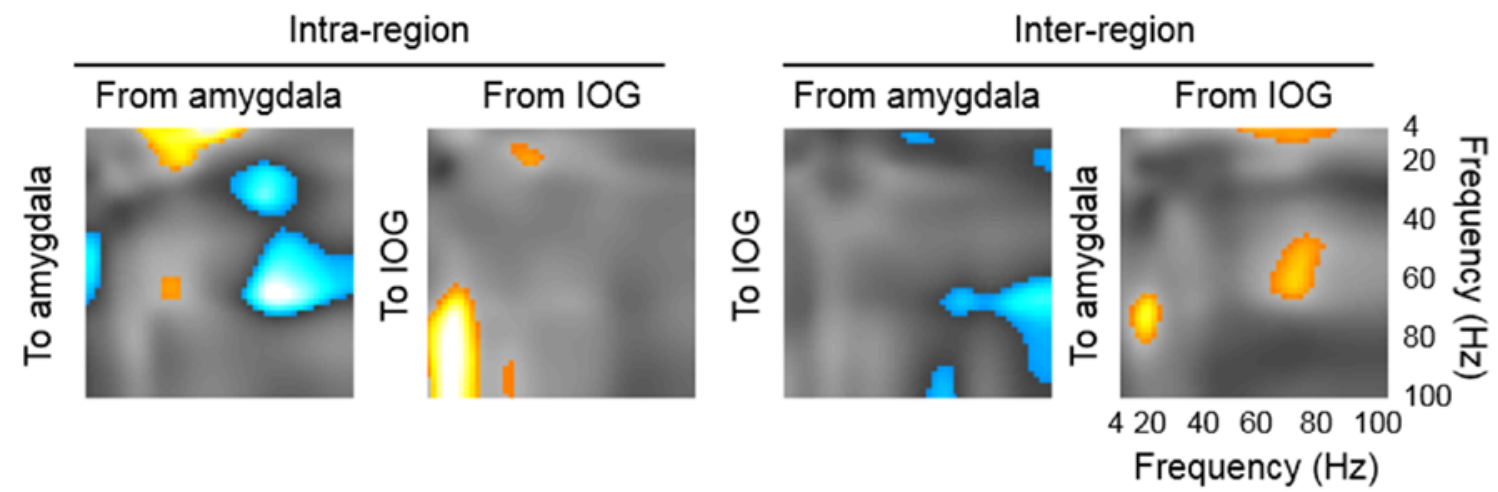

Figure 6. Representative results. (a) Time-frequency maps of right inferior occipital gyrus (IOG) activity for the upright face (FU; left) and upright mosaic (MU; middle) conditions. The SPM $\{T\}$ data for FU versus MU are also shown (right). (b) Functional network models in the IOG and amygdala. Eight possible combinations of modulatory input by FU versus MU onto connections between the IOG and amygdala and self-connection onto each region were investigated. (c) Frequency-frequency modulatory coupling parameters and SPM $\{T\}$ values for FU versus MU of IOG->amygdala and amygdala->IOG modulation are shown. The red-yellow and blue-cyan blobs indicate significant positive/excitatory and negative/inhibitory connectivity, respectively. 